\title{
Development, characterization and test of an ultrasonic vibration-assisted ball burnishing tool
}

\author{
Ramon Jerez-Mesa ${ }^{1 *}$, Jose Antonio Travieso-Rodriguez¹, Giovanni Gomez- \\ Gras $^{1}$, Jordi Lluma-Fuentes ${ }^{2}$
}

\begin{abstract}
${ }^{1}$ Mechanical Engineering Department. Universitat Politècnica de Catalunya. Escola d'Enginyeria de Barcelona Est. Av. d'Eduard Maristany, 10-14, 08019 Barcelona, Spain

2 Material Science and Metallurgical Engineering Department. Universitat Politècnica de Catalunya. Escola d'Enginyeria de Barcelona Est. Av. d'Eduard Maristany, 10-14, 08019 Barcelona, Spain

*Corresponding author: ramon.jerez@upc.edu
\end{abstract}

\begin{abstract}
This paper presents the design and characterization of an ultrasonic vibration-assisted ball burnishing (VABB) tool on Ti-6Al-4V. This process is based on the modification of conventional ball burnishing, by means of the addition of a $40-\mathrm{kHz}$ vibratory force to the burnishing preload exerted by the spring inside the tool. For the purposes of successfully executing the process, a new tool is designed through the assembly of different modules responsible for the various aspects involved in it. That design is hereby presented. Then, a methodology comprising acoustic emission and high frequency sampling is proposed to characterize the functioning of a prototype manufactured according to the previously presented design. The set of techniques deployed to measure the performance of the VABB prototype is presented as a feasible means of characterizing this sort of advanced manufacturing tools, especially like this one which is governed by ultrasonic frequencies. Last of all, the prototype is tested on a Ti-6Al-4V surface to validate it. The superior results of VABB, compared to the non-assisted version of the process, is shown in terms of average and total surface roughness, as well as surface hardness. The highest improvement is achieved by applying the VABB process with $300 \mathrm{~N}$ and 5 passes. The effectiveness of the designed prototype is confirmed, and places this kind of VABB tools as inexpensive systems to successfully execute an innovative finishing process for industrial components.
\end{abstract}

Keywords: vibration-assisted ball burnishing; titanium; hardness; roughness; acoustic emission;

Abbreviations: VABB, vibration-assisted ball burnishing; NVABB, non-vibration-assisted ball burnishing; $A E$, acoustic emission 


\section{Introduction}

Innovation in the manufacturing industry often comes from improving already existing processes, instead of developing brand new operations based on different working principles. One possible way to undertake this innovative approach is based on overlapping an external source of energy that changes the dynamics and/or kinematics of the process. The superposition of vibrations has evidenced positive results to decrease the cutting forces during machining due to the variation of the contact length between tool and material, as explained by Brehl et al. (2008). Vibration assistance has also been reported as a means of enhancing bulk plastic deformation processes, at sight of the works of Mordyuk et al. (2004), who observed metal softening during vibration-assisted drawing of tungsten wires. Huang et al. (2009) confirmed the same effect on copper wires. That softening effect is called acoustoplasticity, which can be described as the decrease of the quasi-static stress required to deform a material as result of the superimposition of a vibratory component on the deforming force (Siu et al., 2001). Ashida and Aoyama (2007) also studied the positive influence of vibration assistance on press forming, and proved that it could prevent the cracking of the workpieces due to the decrease in the friction during the process.

At sight of the results presented above, it is clear that the source of the positive effects delivered by vibration-assistance can be experimentally detected, although they can be interpreted from different point of views. Since ball burnishing is a process based on plastic deformation, it is also eligible to be positively assisted by vibrations. That process does not affect the bulk material, but the surface of the target workpiece, by the effect of a ball which rolls over it deforming its texture irregularities (Travieso-Rodriguez et al., 2011). The vibrationassisted ball burnishing (VABB) refers to the incorporation of a vibratory movement on the burnishing ball during the execution of the process. This type of operation has been reported previously in the bibliography under different denominations, executed on different machines, and governed by different process parameters setups, as described in the next paragraph.

Pande \& Patel (1983) attached an electromagnet to a ball burnishing tool installed on a lathe, executing the VABB process assisted by $60 \mathrm{~Hz}$ oscillations of a maximum of $2.5-\mu \mathrm{m}$ amplitude. The results proved that the material hardness increased compared to the nonassisted version of the operation. Later, Bozdana et al. (2005) and Bozdana \& Gindy (2008) 
deployed an alternative setup in a milling machine, by attaching a $20-\mathrm{kHz}$ ultrasonic generator to a hydrostatic ball burnishing tool. The theoretical amplitude (for the unloaded tool), was established at $6.75 \mu \mathrm{m}$. They concluded that lower pressure can be applied on the ball to obtain similar results than through non-vibration-assisted ball burnishing (NVABB). Although results in terms of roughness were modest, higher work hardening was achieved on the tested Ti-6Al-4V surfaces. A similar system, based on $20-\mathrm{kHz}$ vibrations delivered by a magnetostrictive converter, was tested on a lathe by Huuki \& Laakso (2013) on tempering steel, and by Huuki et al. (2014) on steel alloys and non-ferrous metals. Results were diverse, but hardness improvement was detected on the surfaces after the VABB process. The same system was used on stainless steel additive manufactured parts by Salmi et al. (2017), with positive results.

A different range of frequencies were tested by Gomez-Gras et al. (2015), by designing and successfully applying a VABB tool assisted by a $2.1-\mathrm{kHz}$ vibration, delivered by alternative deflection of thin plates as a result of an oscillatory magnetic field transmitted by a coil. Travieso-Rodriguez et al. (2015a) tested that tool satisfactorily on G10380 specimens, and A92017-T4 aluminum (Travieso-Rodriguez, 2015b). In both cases, the average surface roughness of vibration-assisted ball burnished specimens was substantially better with regards to the ones burnished with no assistance. However the resulting deep hardness profiles or residual stresses measured trough X-ray diffraction were not affected by the process differently from the way that the conventional ball burnishing process did. Later, Amini et al. (2017) applied the VABB process through $17-23 \mathrm{kHz}$ vibration based on a piezoelectric transducer, adjusted by a FEM, although the authors do not specify the actual frequency applied during the assisted tests, but set the amplitude in $8 \mu \mathrm{m}$. The burnishing force was regulated directly by controlling the depth of penetration of the ball with the help of a dynamometer installed in the milling machine. Aluminum 6061-T6 and AISI 1045 steel alloys were the target materials. Results were highly positive in terms of roughness, hardness and residual stress, in favor of the VABB process. The same prototype has recently been used by Teimouri et al. (2018), at two amplitudes, $5 \mu \mathrm{m}$ and $10 \mu \mathrm{m}$, concluding that the residual hardening after ultrasonic burnishing and residual stress have higher values with an increase of the amplitude values.

The common factor between all the cited VABB references is that tests were undertaken by particular designs. This is obvious, due to the fact that no commercial tool is 
available. However, most of those references do not detail in depth the working mechanisms of the vibration transmission, which is a basic factor to understand how the VABB process can be delivered in practice into the system. Furthermore, the range from $60 \mathrm{~Hz}$ to $20 \mathrm{kHz}$ has been reported, but no data is available for higher frequencies. For that reason, the tool that is tested in this paper has been designed to perform the process with a $40-\mathrm{kHz}$ frequency and $10-\mu \mathrm{m}$ amplitude of the vibratory force.

The interest of this paper is multiple. On one hand, it focuses on implementing innovative tool design for the manufacturing engineering sector, by taking advantage of piezoelectric transducers to assist the ball burnishing process through a $40-\mathrm{kHz}$ ball burnishing prototype. With that aim, the design of a new vibration-assisted ball burnishing (VABB) tool is presented, and materialized in a prototype. The tool's $40-\mathrm{kHz}$ frequency regime poses a high difficulty to be characterized, hence the second main issue tackled in this paper: the validation of different engineering techniques to characterize the prototype behavior. Finally, the effectiveness of the prototype is tested on a Ti-6Al-4V flat surface to assess the surface enhancement delivered by the introduction of a vibratory force over the static preload. Results shall be measured in terms of surface roughness and hardness.

\section{VABB tool development}

For the execution of the ball burnishing process, tooling is highly diverse. Depending on their construction and geometry, ball burnishing tools allow to execute different versions of the process. Some tools are highly versatile, whereas others are specifically designed for unique operations such as burnishing of cylindrical inner surfaces (El-Axir et al., 2007).

Another main source of variation of burnishing tools comes from the way that the burnishing preload $F_{p}$ is regulated. Some tools are based on hydrostatic pressure, that is, using the machine tool lubricant as the hydrostatic bearing on which the burnishing tool rolls freely. Depending on the pressure exerted by an external pump, the burnishing force can be regulated, as describe Travieso-Rodriguez et al. (2011). Spring-compression based burnishing tools are also highly extended, as the force can be easily regulated by compressing a calibrated spring. In this case, the burnishing force is governed by the Hooke's law (El-Taweeel and El-Axir, 2009). 
The tool proposed to execute the VABB process is based on the spring-compression functioning principle. Consequently, in this kind of tools, the burnishing force can be calculated through Eq. (1).

$$
F_{b}=F_{p}+F_{v}+\eta
$$

where $F_{b}$ is the total burnishing force, $F_{p}$ is the preload force, $F_{v}$ is the ultrasonic vibratory force, and $\eta$ are the low frequency force variations due to surface irregularities during the feed movement of the tool, and, therefore, extrinsic to its design.

The VABB tool design proposed in this paper incorporates an innovative system to support the burnishing ball, based on a set of bearing balls embedded inside the tool tip, which acts also as the sonotrode amplifying the vibration caused by the piezoelectric module. To successfully execute the process, the new prototype must comply with the following basic specifications:

- Guarantee the constant rolling of the burnishing ball during the process.

- Control with a certain reliability the burnishing preload magnitude.

- Enable the interchangeability of the tool on different machine tools.

Besides, the tool enabling to execute the VABB process has some additional requirements, as follows:

- Separate all electronic devices from inside the humid machine tool environment.

- Transmit the vibratory movement originated at the piezoelectric thickness alternative change to the burnishing ball.

A design made up of three units, namely, the force regulation, vibrations transmission and force transmission units, is proposed (Fig. 1). Each of them is responsible for the compliance of the different intrinsic functions of a VABB tool. 


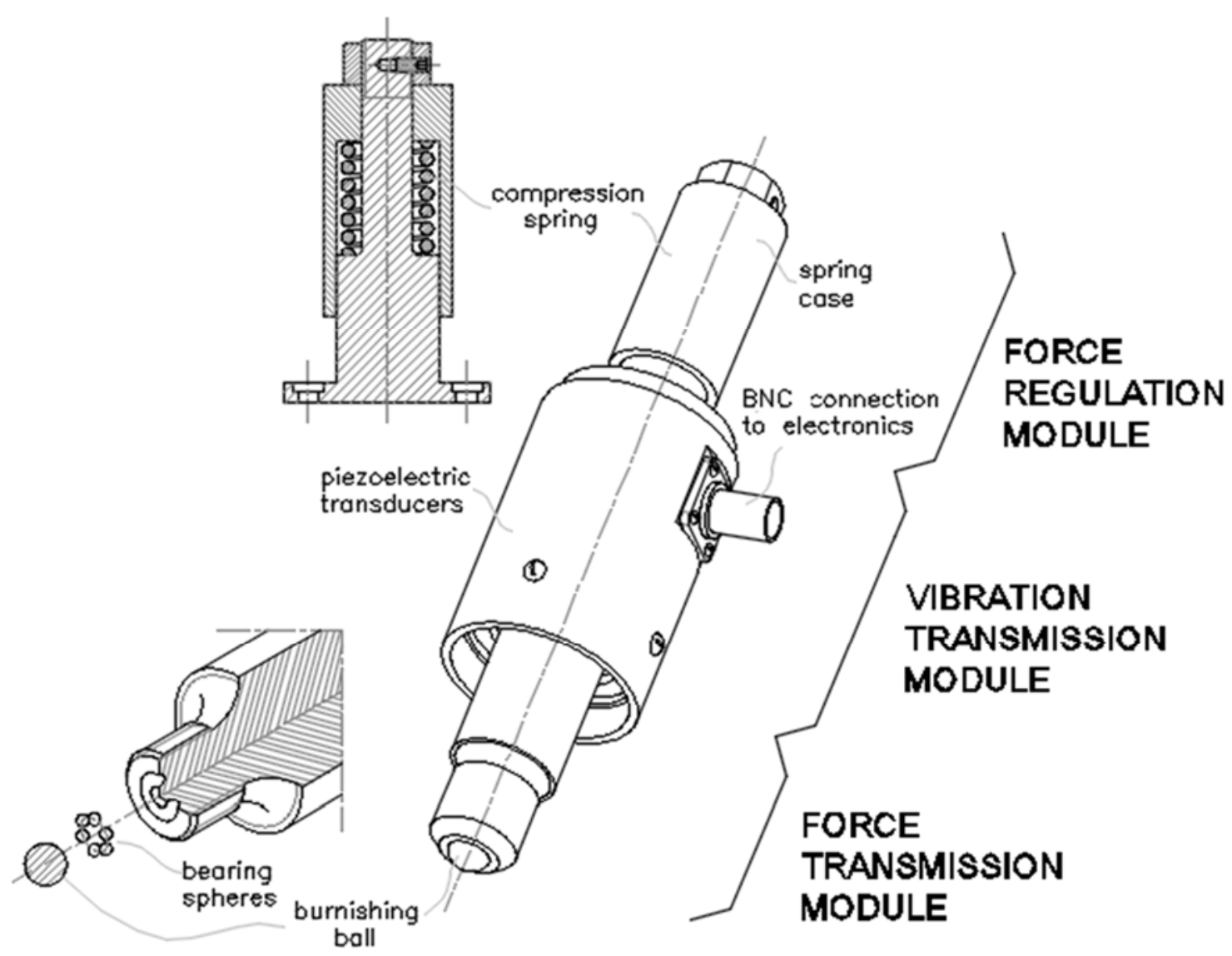

Figure 1. General scheme of the proposed design for the VABB tool.

\subsection{Force regulation unit}

The force regulation unit is composed of the parts responsible for the selection of the preload, $F_{p}$, applied during a ball burnishing operation. It also serves to guarantee the fixation of the tool to the machine. The Hooke's law governing the elastic force applied by the spring contained inside the unit, defines the force level applied during the process. To accurately take profit of this principle, a previous calibration phase must be undertaken to define the actual stiffness and preload of the spring. In effect, the spring is preloaded by the cylinder in which it is housed, and fixed by a screw. That cylinder also serves as the element to be clamped inside the tool holder to attach the tool to the machine-tool. A detail of this setup can be found in Fig. 1.

\subsection{Vibration transmission unit}

This module is composed of the elements necessary to generate the ultrasonic vibratory component of the burnishing force, $F_{v}$. The generation principle of this force is based 
on the fact that the thickness of a polarized piezoelectric material changes when it is subjected to a difference of potential. The equation governing this change of thickness, $\Delta t$, is represented by Eq. (2), and has been deduced from the characteristic electromechanical equations particularized for stacked axial piezoelectric disks (Arnau, 2004), as is the case.

$$
\Delta t=n \cdot d_{33} \cdot V
$$

where $n$ is the number of stack shells, $d_{33}$ is the piezoelectric charge coefficient corresponding to the piezoelectric widening along the longitudinal direction of the tool, and $V$ is the applied voltage.

Piezoelectric transducers are able to follow high frequency electrical excitation, so that they shall deform accordingly at the same frequency as the electrical field. For that reason, a stacked piezoelectric transducer is the main component of this unit. It is contained inside an enclosed case along with the corresponding counterweight. Screwed at the top of that case is the spring support, and, therefore, the whole force regulation unit. The electrical excitation of the vibration transmission unit is achieved by the connection of the electronic circuit with the electrodes between the piezoelectric disks by means of an external BNC plug (Fig. 2).

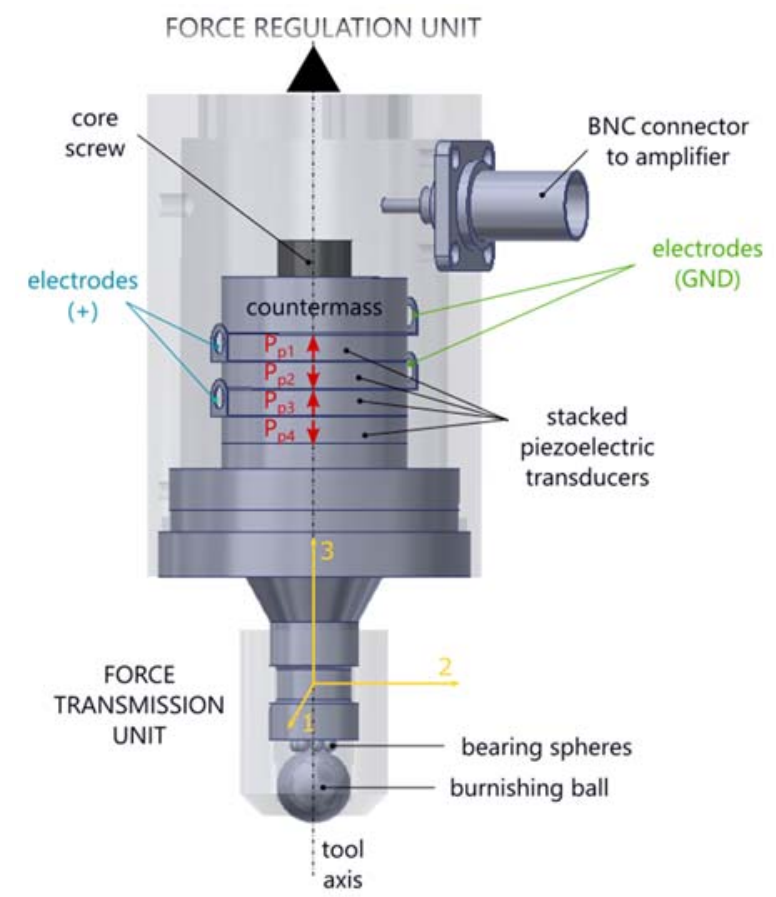

Figure 2. Detail of the piezoelectric stack inside the vibrations transmission unit joint to the force transmission unit. 


\subsection{Force transmission unit}

This function assigned to this module is executing the burnishing force, $F_{b}$, by transmitting both the preload and the vibratory forces generated at the previously described units. The ultimate mechanical transmitter of that force is the burnishing ball, which is supported on a set of bearing spheres inside a hemispherical slot machined on the tool head. That head is actually the extension of the thin support surface on which the piezoelectric disks are fixed. It acts as the sonotrode amplifying the piezoelectric vibration, to the burnishing ball. This technical solution can be observed in Fig. 2 .

\section{VAAB prototype characterization}

The previously described design was implemented by manufacturing a VABB prototype. The piezoelectric module was materialized through a stack composed of 4 hard PZT8 disks. The properties of this piezoelectric ceramic can be consulted in Table 1. The electrical circuit was conceived to excite the transducers with a sinusoidal $40-\mathrm{kHz}$ electrical signals, delivering a maximum voltage of $1.3 \mathrm{kV}$. Consequently, according to Eq. (2), the maximum piezoelectric thickness variation is of $1.5 \mu \mathrm{m}$. The FTU acts as the sonotrode responsible for the amplification of the ultrasonic motion up to $8.5 \mu \mathrm{m}$ at the tip of the ball, according to a numerical model developed by the manufacturer. An important component of the system is the electrical circuit, which excites the piezoelectric stacked, and is equipped with a potentiometer that allows the regulation of the maximum difference of potential applied to the piezoelectric stack. By using this switch, a continuous range of piezoelectric amplitudes can be controlled by the user, so that when the voltage is reduced, so is the amplitude of the piezoelectric, and, therefore, the vibration amplitude, as was shown in Eq. (2). The amplitude is coded in this paper in percentage, as it is the graduation available in the circuit controls. In this case, $100 \%$ corresponds to the maximum piezoelectric amplitude, $8.5 \mu \mathrm{m}$.

Table 1. Properties of hard PZT8 material used to manufacture the piezoelectric actuator.

\begin{tabular}{|c|c|c|}
\hline Property & Value & Units \\
\hline$\rho$ & 7700 & $\mathrm{~kg} / \mathrm{m}^{3}$ \\
\hline$E$ & 63 & $\mathrm{MPa}$ \\
\hline$d_{33}$ & $120 \cdot 10^{-12}$ & $\mathrm{~m} / \mathrm{V}$ \\
\hline
\end{tabular}


All the described design specifications must be proved experimentally to characterize the process. However, such a high frequency and low amplitude vibratory behavior posed a remarkable technical challenge. Hence the need of deploying a set of different experimental tests to register and compose the wave generated by the prototype.

\subsection{Acoustic emission}

At sight of the described measurement challenges, an acoustic emission (AE) experimental setup was proposed to evaluate the dynamic behavior of the prototype. $A E$ sensors are devices whose functioning is based on the inverse piezoelectric effect, as they transform a mechanical stimulus into a difference of potential, registered by the proper acquisition system. The range of frequencies typically eligible to detect through $A E$ goes from the acoustic to the ultrasonic frequency range, depending on the sensor and its resonant frequency. The issue with this technique is that it requires high sampling rates of the $\mathrm{MHz}$ order of magnitude. Therefore, it is usually applied for non-continuous measurement, which prevents to manage great data volumes. A typical application of $A E$ is to register transient high-frequency waves, for instance, to detect and monitor of elastic waves originated at subtle changes in materials lattice, such as microcrack nucleation (Martinez-Gonzalez, 2015).

\subsubsection{AE experimental setup}

Albeit the data processing handicap presented by $\mathrm{AE}$ in continuous measurement, an $\mathrm{AE}$ experimental test was undertaken to monitor the signal transmitted from the vibrating tool inside an AISI 1038 block. The workpiece was mounted on a vise of a LAGUN MC600 milling machine, and was face milled to generate a surface perpendicular to the burnishing tool axis. Then, a Vallen VS-150M acoustic emission piezoelectric transducer was fixed through a geltype couplant. It was connected to a Vallen EAP4 $34 \mathrm{~dB}$ pre-amplifier for signal conditioning and then to a Vallen AMSY-5 acquisition system for signal recording. The recorded signals were processed through a Matlab® script through which the signals were filtered and represented in the time and frequency domains. 
Different conditions, shown in Table 2, were the tested. Both burnishing lines and points tests were performed. That way, a possible influence of the feed motion is to be studied. Also, two preload levels where applied on the testing part. A preliminary set of experiments revealed sensor saturation when the $100 \%$ amplitude allowed by the electrical system is selected through the potentiometer. Therefore, only measurements at $25 \%$ and $50 \%$ were registered.

Table 2. Tested conditions and results related to the first peak observed at the frequency spectrum of the transmitted signals acquired through acoustic emission.

\begin{tabular}{|c|l|l|l|l|}
\hline Test & $\boldsymbol{F}_{\boldsymbol{p}} \mathbf{( N )}$ & $\boldsymbol{A}(\mathbf{\%})$ & $\mathbf{f}_{\mathbf{0}}(\mathbf{k H z})$ & $\mathbf{A}_{\mathbf{0}}(\mathbf{m V} \mathbf{)}$ \\
\hline Burnishing & 90 & 0 & 0 & 0 \\
\cline { 2 - 5 } point & 90 & 25 & 38.09 & 70.10 \\
\cline { 2 - 5 } & 90 & 50 & 38.09 & 63.80 \\
\cline { 2 - 5 } & 270 & 0 & 0 & 0 \\
\cline { 2 - 5 } & 270 & 25 & 38.09 & 55.85 \\
\cline { 2 - 5 } & 270 & 50 & 38.09 & 64.88 \\
\hline \multirow{4}{*}{$\begin{array}{c}\text { Burnishing } \\
\text { line }\end{array}$} & 90 & 0 & 0 & 0 \\
\cline { 2 - 5 } & 90 & 25 & 38.09 & 69.00 \\
\cline { 2 - 5 } & 90 & 50 & 38.09 & 61.99 \\
\cline { 2 - 5 } & 270 & 0 & 0 & 0 \\
\cline { 2 - 5 } & 270 & 25 & 38.09 & 52.13 \\
\cline { 2 - 5 } & 270 & 50 & 38.09 & 69.18 \\
\hline
\end{tabular}

\subsubsection{AE results}

The frequency spectrums represented for all registered conditions evidence the exact same first main frequency of the transmitted wave, which is $38.09 \mathrm{kHz}$, as show the results included in Table 2. The signal amplitudes are not representative of the tool's signal intensity, as they depend on several factors, such as the distance from the test to the AE sensor, which were not a controlled during the test.

As an example, Fig. 3 represents the recorded signals and their frequency spectrum, of two burnishing line tests at the same preload level $270 \mathrm{~N}$. Fig. $3 \mathrm{~A}$ shows that during the nonvibration assisted test (that is, when the piezoelectric is not excited), no signal is registered. On the contrary, the $50 \%$ amplitude signal (Fig. 3B) exhibits that the transmission of an ultrasonic wave through the material is composed of a $38.09 \mathrm{kHz}$ signal and successive harmonics. The same behavior is detected in the rest of the tests, which confirms that the signal generated by 
the piezoelectric is stable in frequency, and does not depend on the spring precompression length or the amplitude of the vibration of the piezoelectric.
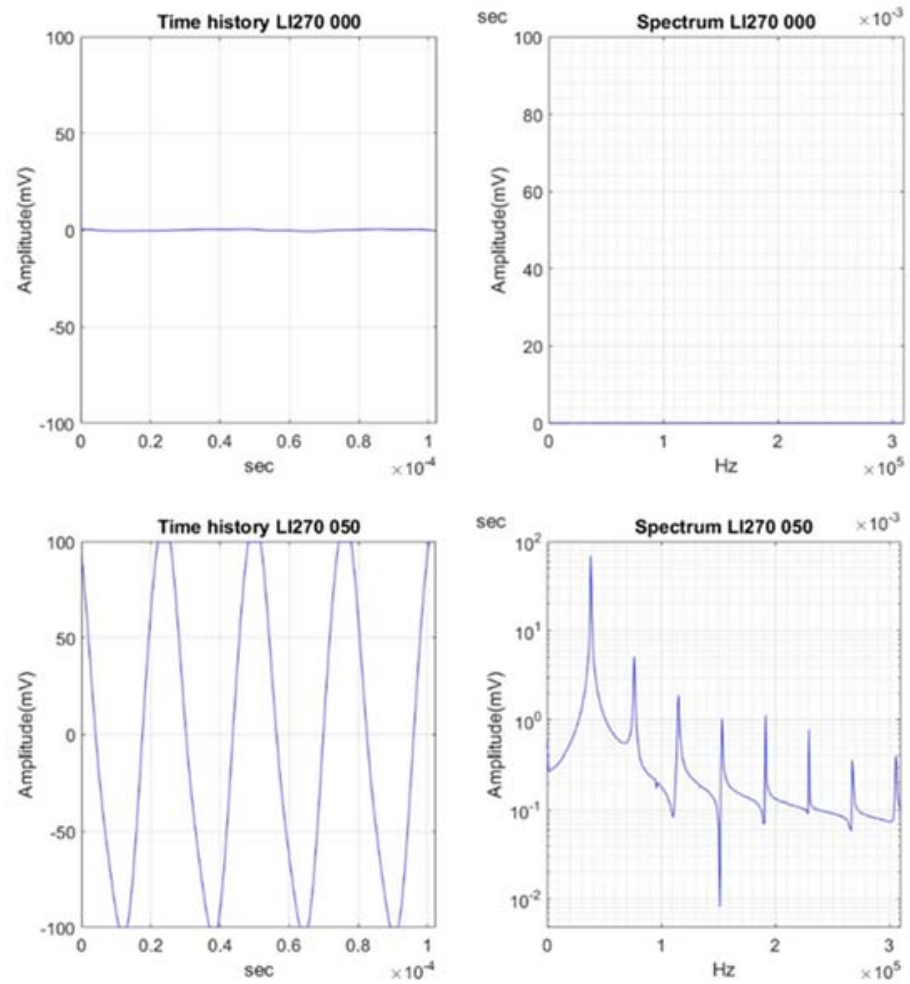

Figure 3. Registered signals for the burnishing line tests applying a $270 \mathrm{~N}$ preload, and $50 \%$ amplitude $V A B B$ and no vibration.

\subsection{Dynamometric characterization}

Acoustic emission proved to validate the frequency of the signal transmitted by the piezoelectric, but could not give evidence of the intensity of that signal. That intensity is directly related to the amplitude of the ball displacement, which in turn defines the vibratory force. As the vibrations amplitude cannot be measured, an alternative second experimental setup using a load cell connected to a high-frequency sampling oscilloscope was deployed.

\subsubsection{Dynamometric experimental setup}

A Kistler 9323A press force sensor was installed at the milling machine vise, and was connected to a Kistler 5015A Charge Meter. A Hameg HM2008 oscilloscope allowed to register the voltage variation detected by the sensor. For a proper reconstruction of the signal, a 10$\mathrm{MHz}$ sampling frequency was used to process the signal by the oscilloscope software. This setup based on sampling through the oscilloscope allowed to sample and reconstruct the signal at a satisfactory frequency. However, it provided a technical limitation linked to the fact that only 
a small time window could be exported, and a continuous measurement was not possible. In order to have a good representativeness of the force, each signal was acquired ten times, and processed by a Matlab $®$ routine to calculate a sinus function fit to model the vibratory force, as exemplifies Fig. 4.



Figure 4. Registered signal and sinus fit for $600 \mathrm{~N}$ and a $100 \% \mathrm{~A}$.

A first set of measurements were taken to construct a calibrating line by progressively compressing the spring inside the prototype, and monitoring the static force. During the second phase, different preload levels and amplitudes were combined and registered. Pre-loads were managed by compressing according to the calibrating function deduced from the previous phase. They resulted from combining 6 levels of the preload with 3 amplitude levels, as can be seen in Table 3.

Table 3. Frequency results of sine fitting curves for all preload and amplitude levels tested during the dynamometric measurements.

\begin{tabular}{|c|l|c|c|}
\hline \multicolumn{2}{|c|}{$\begin{array}{c}\text { Testing } \\
\text { conditions }\end{array}$} & \multicolumn{2}{c|}{ (kHz) } \\
\hline \multirow{2}{*}{$\boldsymbol{F}_{\boldsymbol{p}}(\mathbf{N})$} & $\boldsymbol{A}(\%)$ & Mean & $\begin{array}{c}\text { Standard } \\
\text { deviation }\end{array}$ \\
\hline 100 & 10 & 39.19 & 25 \\
\cline { 2 - 4 } & 50 & 39.18 & 17 \\
\cline { 2 - 4 } & 100 & 39.15 & 25 \\
\hline \multirow{3}{*}{200} & 10 & 39.17 & 12 \\
\cline { 2 - 4 } & 50 & 39.18 & 13 \\
\cline { 2 - 4 } & 100 & 39.17 & 8 \\
\hline 300 & 10 & 39.17 & 8 \\
\cline { 2 - 4 } & 50 & 39.16 & 14 \\
\cline { 2 - 4 } & 100 & 39.16 & 18 \\
\hline
\end{tabular}




\begin{tabular}{|l|l|c|c|}
\hline 400 & 10 & 39.18 & 12 \\
\cline { 2 - 4 } & 50 & 39.17 & 11 \\
\cline { 2 - 4 } & 100 & 39.17 & 17 \\
\hline 500 & 10 & 39.18 & 11 \\
\cline { 2 - 4 } & 50 & 39.17 & 11 \\
\cline { 2 - 4 } & 100 & 39.17 & 9 \\
\hline 600 & 10 & 39.17 & 11 \\
\cline { 2 - 4 } & 50 & 39.17 & 15 \\
\cline { 2 - 4 } & 100 & 39.17 & 23 \\
\hline
\end{tabular}

For each condition, the tool was compressed directly on the sensor, until the objective preload value was achieved. Then, the measurement was reset, and the piezoelectric was excited. That way, only the additional vibratory force due to the piezoelectric actuation was registered by the sensor.

\subsubsection{Quantification of the superimposed vibratory force}

The Matlab® routine allowed to calculate the sine fitted function of the vibratory force, $F_{v}{ }^{*}$, according to the structure shown at Eq. (3).

$$
F_{v}{ }^{*}=\Delta F_{v} \sin (\omega t+\varphi)
$$

where $\Delta F_{v}$ is the vibratory force variation in one half cycle, $\omega$ is the angular frequency, and $\varphi$ is the phase.

All measuring conditions exhibited a $R^{2}$ Pearson coefficient higher than $95 \%$, proving the sinusoidal nature of the vibratory force. Furthermore, frequencies transformed into $\mathrm{Hz}$ exhibited that the sine signals for all testing conditions had a $39.2 \mathrm{kHz}$ (Table 3), slightly higher than the one found with acoustic emission, but consistently constant throughout the tests regardless of the preload.

The real extreme points recorded by the acquisition system in both the negative and the positive half cycles $\left(F_{v, \min }\right.$ and $F_{v, \max }$ respectively) were retained, and graphically represented along with the sine fitting parameters $\Delta F_{v}$ at Fig. 5. All preload forces, for all external amplitudes, show the same average force increase in a half cycle, around $\pm 3 \mathrm{~N}$, except for the $200 \mathrm{~N}$ preload force, where a slightly higher value of $4.5 \mathrm{~N}$ is observed. It can be explained due to the fact that the stiffness of the mechanical system changes with the preload, being minimum at a preload of $200 \mathrm{~N}$, thus amplifying the signal. In principle, this difference of additive force 
depending on the preload should not be influential in the result, as both values are in relative terms similar.
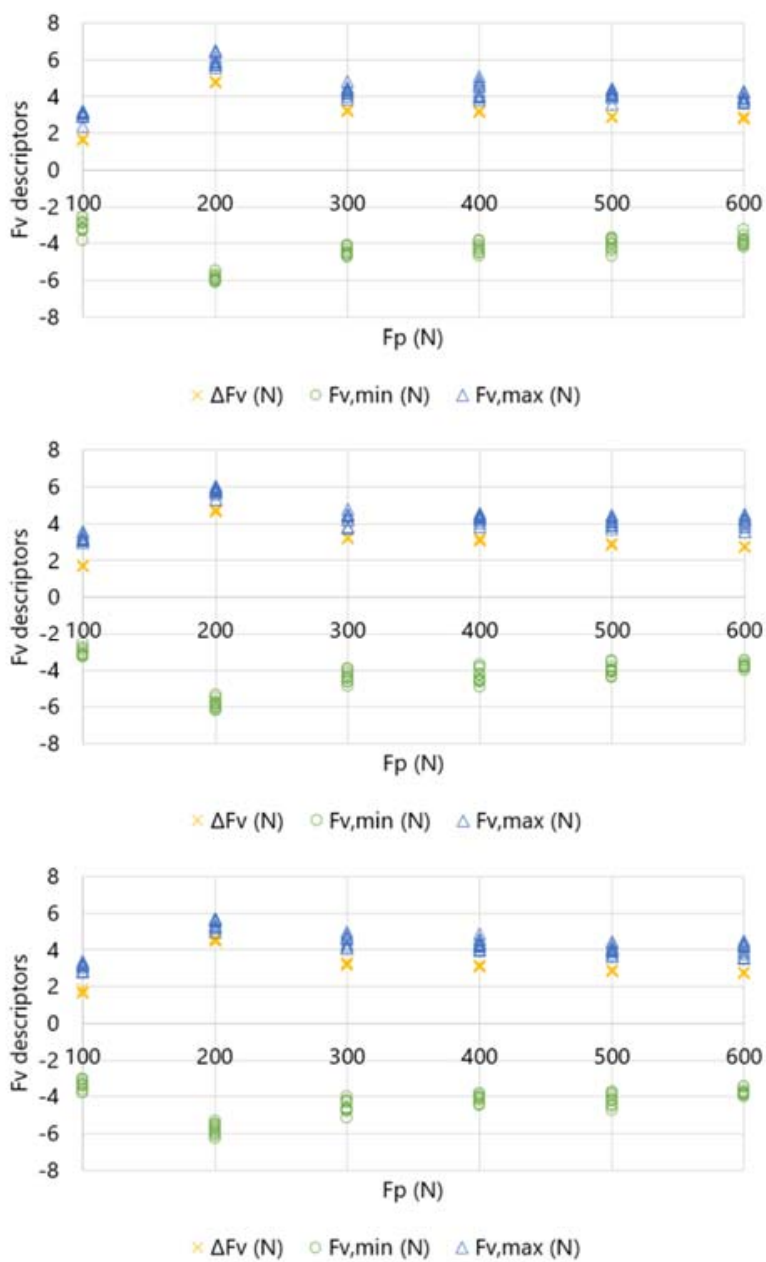

Figure 5. Half cycle vibratory force variation and extreme values for different preload levels.

\section{Prototype preliminary testing}

The VABB prototype was tested on a grade 23 Ti-6Al-4V surface, by applying a complete factorial experimental design in which the vibrations amplitude, the burnishing preload and the number of passes were tested at different levels, resulting a 12-run experimental matrix (Table 4). The workpiece was fixed in the vise, and surfaced with a $\varnothing 80$ front mill with 5 inserts. A $60 \mathrm{~m} / \mathrm{min}$ cutting velocity and a feed of $60 \mathrm{~mm} / \mathrm{min}$ were selected for the operation, as values typically used to perform a finishing pass on this kind of titanium alloy. Then, the VABB prototype was attached at the milling machine through the tool holder, and connected to the external circuit (Fig. 6). For each experimental run, a $10 \times 10 \mathrm{~mm}$ burnishing patch was 
performed along the milling direction, and taking a constant 0.2-mm lateral pass width between adjacent passes.

Table 4. Factors and levels included in the factorial experimental design to test the prototype

\begin{tabular}{|c|c|c|c|}
\hline Factor & Level 1 & Level 2 & Level 3 \\
\hline $\boldsymbol{A} \mathbf{( \% )}$ & 0 & 50 & 100 \\
& NVABB & VABB & VABB \\
\hline $\boldsymbol{F}_{\boldsymbol{p}} \mathbf{( N )}$ & 90 & 270 & - \\
\hline $\boldsymbol{n}_{\boldsymbol{p}}$ & 1 & 5 & - \\
\hline
\end{tabular}



Figure 5. Experimental setup to perform the ball burnishing tests.

To evaluate the results, three variables were evaluated, namely, both the average and total surface roughness perpendicular to the burnishing direction, and the Vickers hardness on the surface. The roughness measurements were taken with a Mitutoyo SJ-210 Surftest profilometer with a cutoff or sampling length of $0.8 \mathrm{~mm}$, and an evaluation length composed of 5 times that sampling length, according to the DIN EN ISO 3274 standard implemented in the device calculation software. To guarantee repeatability in the results, and that the roughness measurements were representative of the overall patch roughness, 20 measurements were taken and averaged.

Surface hardness was measured with a Buehler 5114 durometer, applying a load of $0.005 \mathrm{~g}$. This load was decided after performing several indentations with different loads during 
a preliminary phase. It was selected so that the indentation was superficial enough to represent purely the effects of the VABB process. For each burnishing patch, 20 hardness measures were taken. Outliers were eliminated from the samples by applying the Chauvenet's criterion.

Surface roughness and hardness results are represented at Table 5. All average roughness and hardness results representing each burnishing condition were subjected to an ANOVA to evaluate the mean effect of each factor, along with second level interactions. Results are presented at the next subsections.

Table 5. Surface roughness and hardness results for all tested conditions in the experimental design.

\begin{tabular}{|c|c|c|c|c|c|c|c|c|}
\hline \multirow{2}{*}{\multicolumn{3}{|c|}{$\begin{array}{l}\text { Testing } \\
\text { conditions }\end{array}$}} & \multicolumn{4}{|c|}{ Surface roughness } & \multirow{2}{*}{\multicolumn{2}{|c|}{ HV50 }} \\
\hline & & & \multicolumn{2}{|c|}{$R_{a}(\mu \mathrm{m})$} & \multicolumn{2}{|c|}{$R_{t}(\mu \mathrm{m})$} & & \\
\hline $\begin{array}{c}A \\
(\%)\end{array}$ & $\begin{array}{l}F_{p} \\
(\mathrm{~N})\end{array}$ & $n_{p}$ & Mean & $\begin{array}{l}\text { Standard } \\
\text { deviation }\end{array}$ & Mean & $\begin{array}{l}\text { Standard } \\
\text { deviation }\end{array}$ & Mean & $\begin{array}{l}\text { Standard } \\
\text { deviation }\end{array}$ \\
\hline 0 & 100 & 1 & 0.378 & 0.0234 & 2.502 & 0.1235 & 414 & 11 \\
\hline 0 & 100 & 5 & 0.268 & 0.0314 & 1.759 & 0.1247 & 461 & 12 \\
\hline 0 & 300 & 1 & 0.313 & 0.0143 & 2.249 & 0.2152 & 482 & 9 \\
\hline 0 & 300 & 5 & 0.264 & 0.0342 & 1.659 & 0.1245 & 482 & 12 \\
\hline 50 & 100 & 1 & 0.374 & 0.0124 & 2.567 & 0.0997 & 432 & 14 \\
\hline 50 & 100 & 5 & 0.251 & 0.0091 & 1.702 & 0.1020 & 491 & 8 \\
\hline 50 & 300 & 1 & 0.234 & 0.0145 & 1.587 & 0.1201 & 510 & 16 \\
\hline 50 & 300 & 5 & 0.241 & 0.0245 & 1.502 & 0.1123 & 544 & 12 \\
\hline 100 & 100 & 1 & 0.387 & 0.0412 & 2.654 & 0.2031 & 464 & 11 \\
\hline 100 & 100 & 5 & 0.249 & 0.0189 & 1.658 & 0.1687 & 491 & 9 \\
\hline 100 & 300 & 1 & 0.228 & 0.0203 & 1.600 & 0.1748 & 532 & 14 \\
\hline 100 & 300 & 5 & 0.243 & 0.0247 & 1.499 & 0.1347 & 558 & 14 \\
\hline \multicolumn{3}{|c|}{ Initial Surface } & 0.634 & 0.0347 & 4.417 & 0.2101 & 400 & 9 \\
\hline
\end{tabular}

\subsection{Surface roughness results}

All burnishing tests proved resulted in the decrease of roughness parameters that characterized the initially milled surface. In absolute terms, the minimum average and total roughness parameters were achieved by applying $300 \mathrm{~N}$ and 5 passes, and assisting the process with the maximum piezoelectric amplitude. The average roughness was improved by a $61.6 \%$ ratio, whereas total roughness improved by $66.1 \%$. The results in absence of vibrations while applying the same preload and number of passes show lower improvement ratios, of 
$37.1 \%$ and $49.1 \%$ respectively. Therefore, the introduction of vibrations has a significant impact on roughness reduction, and the VABB proves to have a significant impact on the surface. In the following subsections, results are statistically analyzed.

\subsubsection{ANOVA results. Response variable: average surface roughness}

To analyze the mean effects of each factor on the roughness responses, an analysis of variance (ANOVA) was calculated. Results show an agreement with the results discussed previously, as an increasing preload and number of passes cause a lower average surface roughness (Fig. 7A). The main effects graph shows a second important result, which is that there is the significant roughness reduction achieved by the assisted process, is independent of the piezoelectric amplitude selected by the power circuit. Therefore, the piezoelectric amplitude parameter is not influential, and roughness results are robust regardless of the value of that parameter.

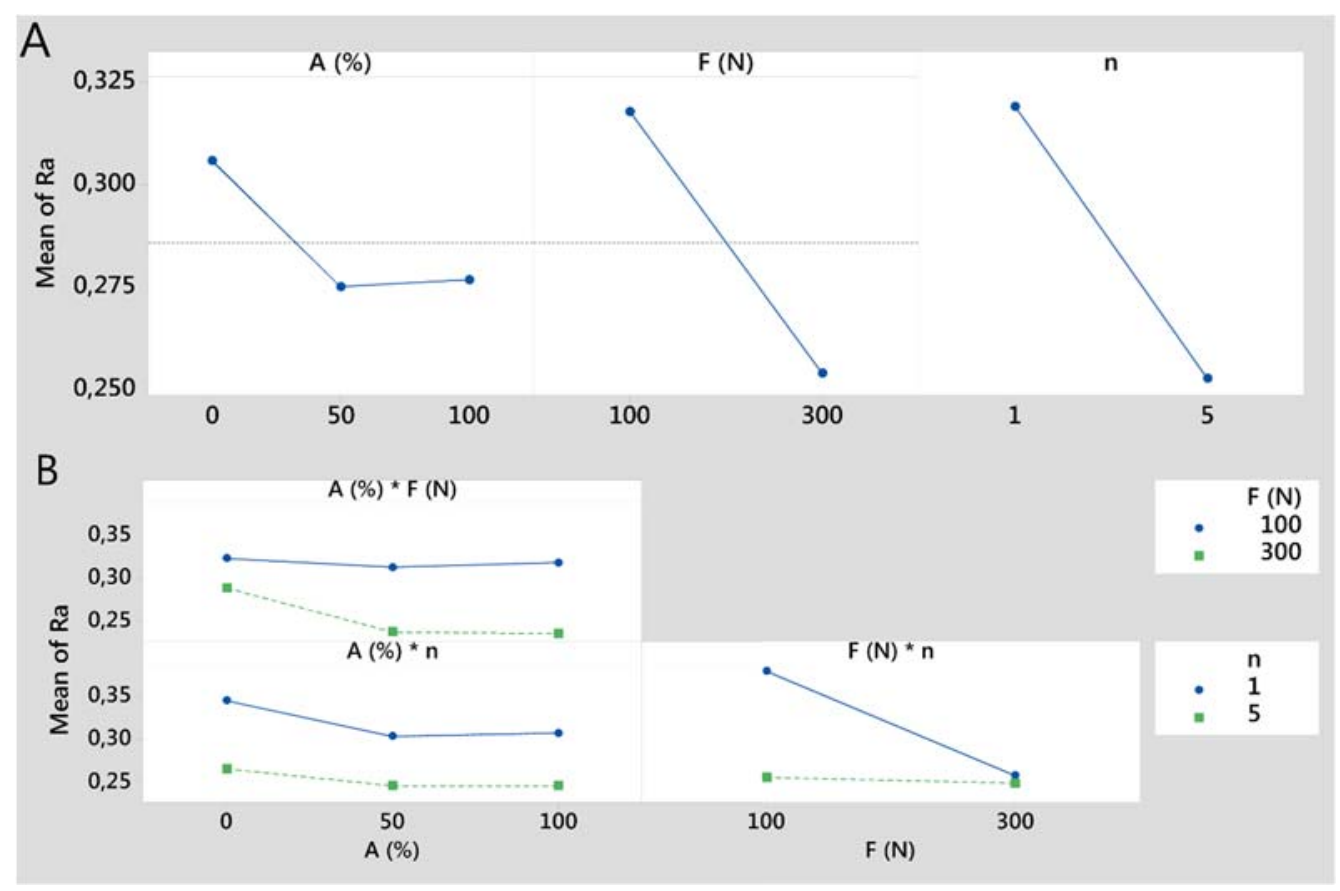

Figure 7. Mean effect plots derived from ANOVA results. Response variable: $R_{a}$. A. First order effects. B. Second order interactions among factors.

Second order effects have also been analyzed (Fig. 7B). A certain interaction between force and number of passes can be seen, as increasing from 1 to 5 passes with a $100 \mathrm{~N}$ preload derives in a remarkable average roughness reduction, but is indifferent in the case of applying $300 \mathrm{~N}$. This could be caused by the fact that a $300 \mathrm{~N}$ preload is sufficiently high to 
cause surface roughness reduction in one pass, whereas 1 pass with a $100 \mathrm{~N}$ preload hardly influences surface roughness. This result is fundamental, as it would allow the reduction of processing time by applying 1 pass with $300 \mathrm{~N}$, if only roughness parameters were considered as optimization target.

\subsubsection{ANOVA results. Response variable: total surface roughness}

The studied factors exhibit a similar mean effect on the total roughness of the surface. In effect, Fig. 8A confirms the direct dependency with preload and number of passes, as well as the higher effectiveness of the vibration-assisted process regardless of the piezoelectric amplitude selected. Fig. 8B proves that only the interaction between the preload and number of passes is significant, in the same sense as with the average surface roughness. In mean terms, the average and total roughness are similarly affected by the assisted process.

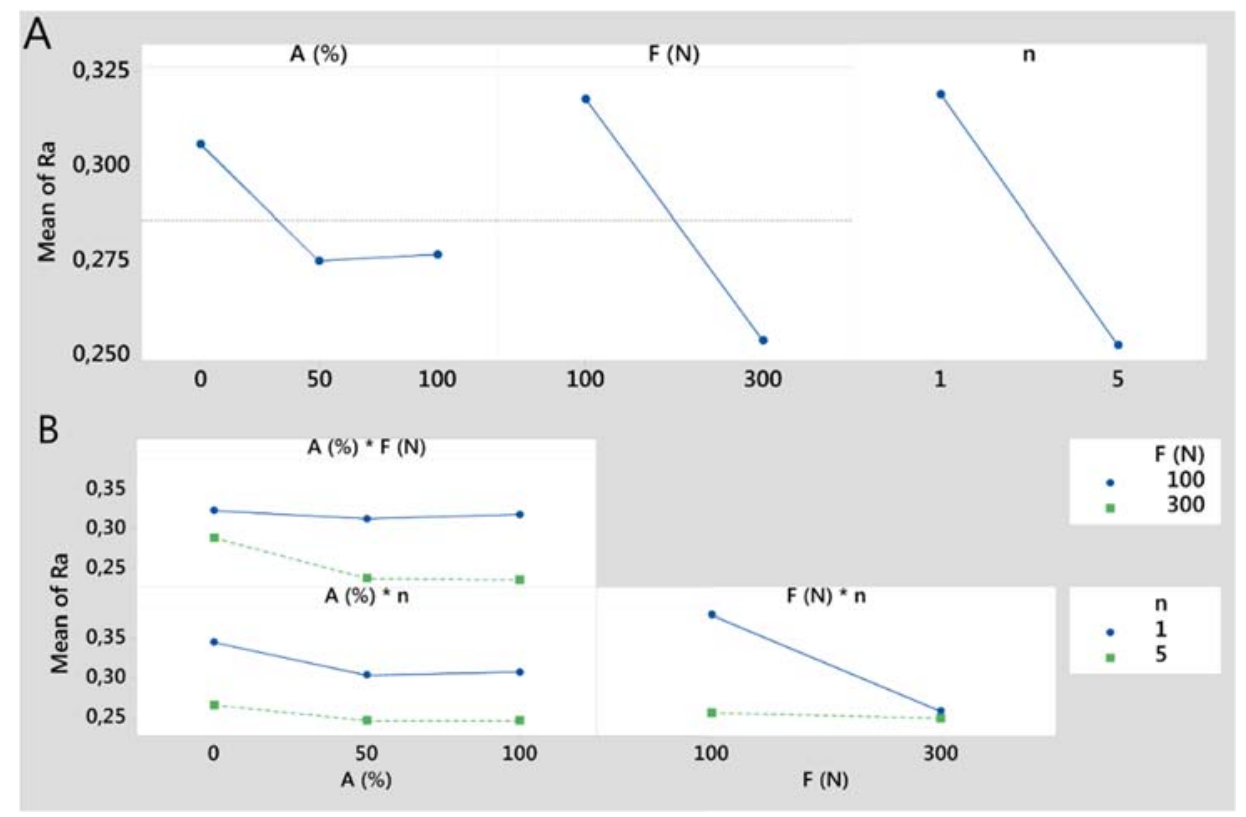

Figure 8. Mean effect plots derived from ANOVA results. Response variable: $R_{t}$. A. First order effects. B. Second order interactions among factors.

\subsection{Surface hardness results}

The highest surface hardness was $558 \pm 14 \mathrm{HV} 0.005$, achieved by applying the VABB process with $300 \mathrm{~N}$ preload and 5 passes. This value corresponds to a $39.5 \%$ improvement ratio with regards to the hardness shown by the initial surface. The NVABB process also achieved the maximum improvement rate by applying the same conditions, but only by $20.5 \%$, confirming the higher effectiveness of the VABB process. 


\subsubsection{ANOVA results. Response variable: surface hardness}

Fig. 9A shows the mean effect plots of each factor on HV0.005 variable. All of them exhibit an influence on hardness results. As the preload and number of passes increase, the surface exhibits higher hardness after burnishing. This is probably caused because of the higher strain hardening experienced by the material as more force and passes are applied on the surface.

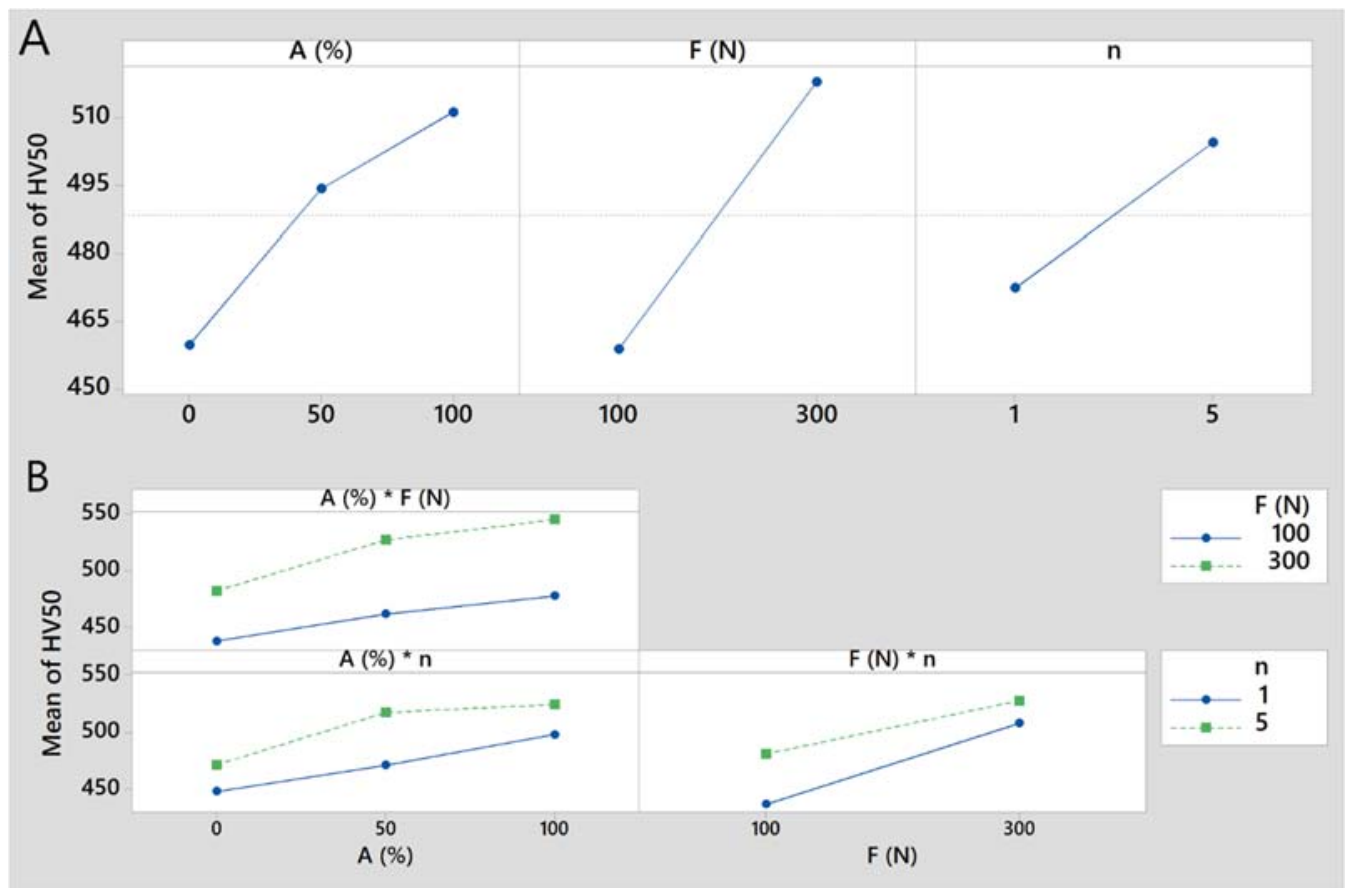

Figure 8. Mean effect plots derived from ANOVA results. Response variable: HV0.005. A. First order effects. B. Second order interactions among factors.

The NVABB process exhibits a considerable lower hardness compared to the VABB one, what confirms the successful results delivered by the prototype object of study at this paper. It can be said that strain hardening achieved by a vibratory force during burnishing is higher than when the process is exclusively performed by applying the preload. On the other hand, there a different hardness between amplitude levels of $50 \%$ and $100 \%$, meaning that an influence of the amplitude is observed in terms of hardness results. To maximize the hardness of VABB surfaces, the maximum amplitude should be applied. This result agrees with the conclusions reached by Temouri et al. (2018), and it can be concluded that the selection of the amplitude change during $\mathrm{VABB}$ is an influential factor to define the residual hardening. In this 
experimental execution, a 8.5- $\mu \mathrm{m}$ amplitude has been found the best value for those purposes. However, it must be considered that Pande \& Patel (1986) observed the same, but found a maximum threshold preload level, so that higher values delivered lower hardness. Therefore, it cannot be known whether amplitudes higher than $8.5 \mu \mathrm{m}$ could also deliver this detrimental interaction with the preload. Neither whether this threshold depends on the material. Both questions should be studied with other systems that allow to reach higher amplitude levels.

On the contrary, Fig. 9B shows that no interaction among factors is observed, so the model relating the evaluated factors in terms of resulting hardness is basically linear. All these ANOVA results were validated by calculating an Anderson-Darling normality test on the residuals of the model, with a p-value lower than $5 \%$, confirming the positive results. The $\mathrm{R}^{2}=$ $98.65 \%$ of the adjusted model also confirms the adequacy of the model to describe the process effects.

\section{Conclusions}

A new prototype has been manufactured and successfully tested to execute the vibration-assisted ball burnishing process. It works based on the vibration of a piezoelectric stack designed to work at $40 \mathrm{kHz}$. Acoustic emission and high frequency acquisition techniques have been successfully applied to characterize the tool, finding descriptive and consistent data that lead to think of the stability of the prototype and the feasibility to be manufactured.

To validate the design, the VABB prototype was successfully tested on Ti-6Al-4V surface through a complete factorial experimental design. All burnishing conditions proved to increase the surface hardness and reduce the average and total surface roughness, with a higher enhancement rate as the preload and number of passes increase. The optimal combination of parameters inside the tested values, considering surface roughness minimization and hardness maximization, can be established in $300-\mathrm{N}$ preload and 5 passes performed with the VABB combined with a $100 \%$ amplitude.

Compared to the non-assisted process, the VABB process showed a higher improvement rate in average surface roughness $(61.6 \%$ in contrast to $37.0 \%)$, total roughness (66.1\% against $49.1 \%)$, and surface hardness (39.5\% in contrast to $20.5 \%)$. The higher hardness shown by the material after VABB, compared to the NVABB results, can be an 
indicator of the higher degree of plastic deformation experienced by the material, as work hardening is related to it. The amplitude of the vibration proved not to be an influential factor in the roughness results. On the contrary, hardness results evidenced that a higher amplitude leads to higher residual hardening. This result implies that the highest amplitude should be selected with this kind of prototype to obtain the best results on Ti-6Al-4V.

\section{Acknowledgements}

We would also like to thank the company CEUS S.L. for their advice on the implementation of the piezoelectric transducer.

\section{Funding}

This study was supported by the Spanish Ministry of Economy and Competitiveness [grant number DPI2015-69803-R], which is greatly appreciated. 


\section{References}

Amini, S., Bagheri, A., Teimouri, R. 2017. Ultrasonic-assisted ball burnishing of aluminum 6061 and AISI 1045 steel. Mater Manuf Proc 1-10. DOI: https://doi.org/10.1080/10426914.2017.1364862

Arnau, A. (Ed.), 2004. Piezoelectric transducers and applications, ed. Springer-Verlag Heidelberg, Berlin.

Ashida, Y., Aoyama, H., 2007. Press forming using ultrasonic vibration. J Mater Process Technol. 187, 118-122. DOI: 10.1016/j.jmatprotec.2006.11.174.

Bozdana, A. T., Gindy, N. N., Li, H. 2005. Deep cold rolling with ultrasonic vibrations-a new mechanical surface enhancement technique. Int J Mach Tool Manu 45(6), 713-718. DOI: https://doi.org/10.1016/j.ijmachtools.2004.09.017

Bozdana, A. T., Gindy, N. N. Z. 2008. Comparative experimental study on effects of conventional and ultrasonic deep cold rolling processes on Ti-6Al-4V. Mat Sci Tech 24(11), 1378-1384. DOI: https://doi.org/10.1179/174328408X302431

Gómez-Gras, G., Travieso-Rodríguez, J. A., González-Rojas, H. A., Nápoles-Alberro, A., Carrillo, F. J., Dessein, G., 2015. Study of a ball-burnishing vibration-assisted process. Proc Inst Mech Eng B. 229(1), 172-177. DOI: 10.1177/0954405414526383.

El-Axir, M. H., Othman, O. M., Abodiena, A. M., 2008. Study on the inner surface finishing of aluminum alloy 2014 by ball burnishing process. J Mater Process Technol. 202(1), 435-442. DOI: 10.1016/j.jmatprotec.2007.10.040.

El-Taweel, T. A., and El-Axir, M. H., 2009. Analysis and optimization of the ball burnishing process through the Taguchi technique. The International Journal of Advanced Manufacturing Technology. 41(3), 301-310. DOI: 10.1007/s00170-008-1485-6.

Huang, H., Pequegnat, A., Chang, B. H., Mayer, M., Du, D., Zhou, Y., 2009. Influence of superimposed ultrasound on deformability of Cu. J Appl Phys. 106(11), 113514. DOI: 10.1063/1.3266170. 
Huuki, J., Laakso, S. V. 2013. Integrity of surfaces finished with ultrasonic burnishing. P I Mech Eng B-J Eng 227(1), 45-53. DOI: https://doi.org/10.1177/0954405412462805

Huuki, J., Hornborg, M., Juntunen, J. 2014. Influence of ultrasonic burnishing technique on surface quality and change in the dimensions of metal shafts. Journal of Engineering, 2014. DOI: http://dx.doi.org/10.1155/2014/124247

Izumi, O., Oyama, K., and Suzuki, Y., 1966. Effects of superimposed ultrasonic vibration on compressive deformation of metals. Transactions of the Japan institute of metals. 7(3), 162-167.

Martinez-Gonzalez, E., Ramirez, G., Romeu, J., and Casellas, D., 2015. Damage induced by a spherical indentation test in tool steels detected by using acoustic emission technique. Exp Mech. 55(2), 449-458. DOI: 10.1007/s11340-014-9959-y.

Mordyuk, B. N., Mordyuk, V. S., Buryak, V. V., 2004. Ultrasonic drawing of tungsten wire for incandescent lamps production. Ultrasonics. 42(1), 109-111. DOI: 10.1016/j.ultras.2004.02.002.

Pande, S. S., Patel, S. M., 1984. Investigations on vibratory burnishing process. Int J Mach Tool D R 24(3), 195-206. DOI: https://doi.org/10.1016/0020-7357(84)90004-0

Salmi, M., Huuki, J., Ituarte, I. F. 2017. The ultrasonic burnishing of cobalt-chrome and stainless steel surface made by additive manufacturing. Progress in Additive Manufacturing, 2(1-2), 3141. DOI $10.1007 / s 40964-017-0017-z$

Siu, K. W., Ngan, A. H. W., Jones, I. P., 2011. New insight on acoustoplasticity-Ultrasonic irradiation enhances subgrain formation during deformation. Int J Plas. 27(5), 788-800. DOI: 10.1016/j.ijplas.2010.09.007.

Teimouri, R., Amini, S., Bami, A. B. 2018. Evaluation of optimized surface properties and residual stress in ultrasonic assisted ball burnishing of AA6061-T6. Measurement 116, 129-139. DOI: https://doi.org/10.1016/j.measurement.2017.11.001

Travieso-Rodríguez, J. A., Dessein, G., González-Rojas, H. A., 2011. Improving the surface finish of concave and convex surfaces using a ball burnishing process. Mater Manuf Processes. 26(12). 1494-1502. DOI: 10.1080/10426914.2010.544819. 
Travieso-Rodriguez, J. A., Gomez-Gras, G., Dessein, G., Carrillo, F., Alexis, J., Jorba-Peiro, J., Aubazac, N., 2015. Effects of a ball-burnishing process assisted by vibrations in G10380 steel specimens. Int J Adv Manuf Technol. 81(9-12), 1757-1765. DOI: 10.1007(s00170-015-7255-3.

Travieso-Rodríguez, J. A., Gras, G. G., Peiró, J. J., Carrillo, F., Dessein, G., Alexis, J., Rojas, H. G., 2015. Experimental study on the mechanical effects of the vibration-assisted ball-burnishing process. Mater Manuf Processes. 30(12), 1490-1497. DOI: 10.1080/10426914.2015.1019114. 